\title{
The total quasi-steady state assumption: its justification by singular perturbation and its application to the chemical master equation
}

\author{
C. F. Khoo ${ }^{1} \quad$ M. Hegland ${ }^{2}$
}

(Received 14 August 2008; revised 18 November 2008)

\begin{abstract}
Deterministic models of enzymatic reactions based on the quasisteady state assumption (QSSA) and total quasi-steady state assumption (tQSSA) have been used successfully in the past. This is surprising as the QSSA and tQSSA can neither be verified mathematically nor by experiment for most cases of interest. Here, we discuss an approach using singular perturbation theory to justify the approximation obtained by tQSSA. In addition, we extend the application of tQSSA to the stochastic model to deal with stiff differential equations originating from the chemical master equation.
\end{abstract}

http://anziamj.austms.org.au/ojs/index.php/ANZIAMJ/article/view/1437 gives this article, (c) Austral. Mathematical Soc. 2008. Published November 23, 2008. ISSN 1446-8735. (Print two pages per sheet of paper.) 


\section{Contents}

1 Introduction

C430

2 Total quasi-steady state approximation

C431

3 Singular perturbation analysis of tQSSA

C432

3.1 Scaling . . . . . . . . . . . . . . . . C433

3.2 Outer solution . . . . . . . . . . . . . . . . . . C434

3.3 Inner solution . . . . . . . . . . . . . . . . C C435

3.4 Matching and uniform approximation . . . . . . . C C436

4 Applying the tQSSA to the chemical master equation

C439

5 Discussion

C441

References

C442

\section{Introduction}

One of the most well-known enzymatic reactions is the so-called MichaelisMenten mechanism:

$$
\mathrm{E}+\mathrm{S} \underset{\mathrm{K}_{-1}}{\stackrel{\mathrm{K}_{1}}{\rightleftharpoons}} \mathrm{C} \stackrel{\mathrm{K}_{2}}{\longrightarrow} \mathrm{E}+\mathrm{R}
$$

which is an irreversible conversion of the substrate, $\mathrm{S}$, into the product, $\mathrm{R}$, through the formation of an intermediate species named complex, $\mathrm{C}$, catalyzed by the enzyme, $\mathrm{E}$. The forward and backward rate constants, here $\mathrm{K}_{1}$, $\kappa_{2}$ and $\kappa_{-1}$, are used for description of the reaction kinetics (see equation (2) and (3)).

Michaelis and Menten [7] proposed that the amount of complex is negligible compared to the amount of substrate in the system (1) where the amount of enzyme present is relatively small compared to the substrate [8]. 
Briggs and Haldane [3] then extended this idea by postulating that the concentration of the complex remains constant in the enzymatic reaction. This approximation is known as the quasi-steady state approximation (QSSA). By utilizing such an assumption, the fast dynamics of the complex are eliminated from the system and thus the model complexity and computational cost are reduced.

\section{Total quasi-steady state approximation}

In some cases, the enzyme concentration is virtually the same or greatly exceeds the substrate concentration in the actual biochemical environment. Thus, the standard QSSA breaks down in the circumstance where there is an excess of enzyme level [2]. In dealing with the invalidity of the QSSA, Borghans and collaborators [2] proposed a new approach, the total quasisteady state approximation (tQSSA), by introducing a lumped variable, the total substrate concentration $[\overline{\mathrm{S}}]=[\mathrm{S}]+[\mathrm{C}]$, to replace the free substrate concentration $[S]$ in classical QSSA. We use $[\mathrm{X}]$ to denote the concentration of species $X$ as a function of time and $X(t)$ to denote the value of this function at time $t$.

The rate equations for the reactions (1) in the tQSSA framework are [2]

$$
\begin{aligned}
\frac{d[\bar{S}]}{d t} & =-\kappa_{2}[C], \\
\frac{d[C]}{d t} & =\kappa_{1}\left(E_{0}-[C]\right)([\bar{S}]-[C])-\left(\kappa_{-1}+\kappa_{2}\right)[C],
\end{aligned}
$$

with initial conditions $\bar{S}(0)=S_{0}$ and $C(0)=0$. The concentrations $[E]$ and $[P]$ are obtained from the conservation laws $[E]+[C]=E_{0}$ and $[S]+[C]+$ $[P]=S_{0}$ where $E_{0}$ is the total enzyme concentration and $S_{0}$ is the total substrate concentration [9]. The association $\left(\kappa_{1}\right)$ and dissociation $\left(\kappa_{2}\right.$ and $\left.\kappa_{-1}\right)$ rate constants carry the units $\mathrm{nM}^{-1} \min ^{-1}$ and $\min ^{-1}$ respectively. 
An inspection of the phase plane for the system of rate equations and further analysis of equations (2) and (3) reveals that a steady state exists at $([\bar{S}],[C])=(0,0)$ and that the complex concentration $[C]$ evolves in two stages:

1. the transient stage or pre-steady state characterised by a rapid increase of $[\mathrm{C}]$;

2. the quasi-steady state during which the complex concentration slowly decays towards the steady state.

In addition, the QSSA and tQSSA postulate that during the pre-steady state the concentrations $[\mathrm{S}]$ (QSSA) and [ $\overline{\mathrm{S}}]$ (tQSSA) are approximately constant [9]. With this assumption, Borghans and collaborators [2] defined two time scales for the system: the fast transient period of the complex in the pre-steady state, $t_{c}$, and the slow time scale for the significant depletion of $[\bar{S}]$ after the transient period, $t_{\bar{s}}$. Specifically, they suggested

$$
t_{c}=\frac{1}{k_{1}\left(E_{0}+S_{0}+\kappa_{m}\right)} \quad \text { and } \quad t_{\bar{s}}=\frac{E_{0}+S_{0}+\kappa_{m}}{\kappa_{2} E_{0}}
$$

where $\kappa_{m}=\left(\kappa_{-1}+\kappa_{2}\right) / \kappa_{1}$ is the Michaelis-Menten constant.

\section{Singular perturbation analysis of tQSSA}

Here we present a mathematical justification for the tQSSA in terms of singular perturbation theory. In particular, we show how to include the initial condition $\mathrm{C}(0)=0$ which cannot be satisfied in the original tQSSA. We have the following steps: 
1. In equations (2) and (3), we scale the variables (see Section 3.1 and 3.2) to yield the equivalent system

$$
\begin{aligned}
& \frac{d s_{O}(T ; \epsilon)}{d T}=f_{1}\left[s_{O}(T ; \epsilon), c_{O}(T ; \epsilon)\right] \\
& \epsilon \frac{d c_{O}(T ; \epsilon)}{d T}=f_{2}\left[s_{O}(T ; \epsilon), c_{O}(T ; \epsilon)\right] .
\end{aligned}
$$

By a theorem of Tikhonov [10], the solution of this system converges to the solution of the differential algebraic equations (DAEs):

$$
\frac{d s_{O}(T ; 0)}{d T}=f_{1}\left[s_{O}(T ; 0), c_{O}(T ; 0)\right], \quad 0=f_{2}\left[s_{O}(T ; 0), c_{O}(T ; 0)\right]
$$

in the outer region as $\epsilon \rightarrow 0$, for example, $s_{O}(T ; \epsilon) \rightarrow s_{O}(T ; 0)$.

2. Rescaling the time differently by setting $\tau=T / \epsilon$ (see Section 3.3), we get another (equivalent) set of equations from system (2) and (3):

$$
\frac{d s_{I}(\tau ; \epsilon)}{d \tau}=\epsilon f_{1}\left[s_{I}(\tau ; \epsilon), c_{I}(\tau ; \epsilon)\right], \quad \frac{d c_{I}(\tau ; \epsilon)}{d \tau}=f_{2}\left[s_{I}(\tau ; \epsilon), c_{I}(\tau ; \epsilon)\right] .
$$

For $\epsilon \rightarrow 0$, this ia a (regularly) perturbed system and one can show that for bounded $\tau, c_{\mathrm{I}}(\tau ; \epsilon) \rightarrow \mathrm{c}_{\mathrm{I}}(\tau ; 0)$ and $\mathrm{s}_{\mathrm{I}}(\tau ; \epsilon) \rightarrow \mathrm{s}_{\mathrm{I}}(\tau ; 0)$ where $\mathrm{c}_{\mathrm{I}}(\tau ; 0)$ and $\mathrm{s}_{\mathrm{I}}(\tau, 0)$ solve

$$
\frac{d s_{I}(\tau ; 0)}{d \tau}=0, \quad \frac{d c_{I}(\tau ; 0)}{d \tau}=f_{2}\left[s_{I}(\tau ; 0), c_{I}(\tau ; 0)\right]
$$

3. The outer and inner solutions are matched at the edge of the boundary layer (see Section 3.4).

\subsection{Scaling}

For the two time periods we introduce two differently scaled dimensionless times:

$$
\tau=\frac{t}{t_{c}} \quad \text { for the transient period; }
$$




$$
\mathrm{T}=\frac{\mathrm{t}}{\mathrm{t}_{\overline{\mathrm{S}}}} \text { for the post-transient period. }
$$

Likewise, the complex and total substrate concentrations are scaled by dividing them by their respective maxima:

$$
c=\frac{[C]}{C_{0}} ; \quad s=\frac{[\bar{S}]}{S_{0}} .
$$

The maximum of $[\mathrm{C}], \mathrm{C}_{0}$, is derived by Borghans et al. [2] as

$$
\mathrm{C}_{0}=\mathrm{E}_{0} \mathrm{~S}_{0} /\left(\mathrm{E}_{0}+\mathrm{S}_{0}+\mathrm{K}_{\mathrm{m}}\right) .
$$

\subsection{Outer solution}

Borghans et al. [2] found a necessary condition for the validity of the tQSSA, namely $t_{c} \leq t_{\bar{S}}$, or alternatively, that $0<\epsilon \leq 1$ where

$$
\epsilon=\frac{t_{c}}{t_{\bar{s}}}=\frac{\kappa_{2} E_{0}}{\kappa_{1}\left(E_{0}+S_{0}+\kappa_{m}\right)^{2}} .
$$

This term is thus used as a small dimensionless parameter in the singular perturbation analysis.

In the outer region, equation (3) is nondimensionalized with the scaled variables $\mathrm{c}$ and $\mathrm{T}$ to give

$$
\begin{aligned}
\frac{d c}{d T} & =\frac{1}{C_{0}} \times k_{1}\left[E_{0} S_{0} s-\left(E_{0}+S_{0} s+\kappa_{m}\right) C_{0} c+\left(C_{0} c\right)^{2}\right] \times t_{\bar{s}} \\
& =\frac{1}{\epsilon}\left(s-\frac{E_{0}+S_{0} s+\kappa_{m}}{E_{0}+S_{0}+\kappa_{m}} c+\gamma c^{2}\right),
\end{aligned}
$$

with

$$
\gamma=\frac{E_{0} S_{0}}{\left(S_{0}+K_{m}+E_{0}\right)^{2}},
$$


and initial condition $\mathrm{c}(0)=0$. As $\epsilon \rightarrow 0$, equation (5) becomes the algebraic equation

$$
s-\frac{E_{0}+S_{0} s+K_{m}}{E_{0}+S_{0}+K_{m}} c+\gamma c^{2}=0 .
$$

By applying the approach discussed by Cha and Cha [5] or Padé approximation [1], an approximation $\mathfrak{c}_{\mathrm{O}}$ (subscript ' $\mathrm{O}$ ' here denotes the outer solution) for $\mathrm{c}$ is obtained:

$$
c_{O}(T)=\frac{E_{0}+S_{0}+\kappa_{m}}{E_{0}+S_{0} s+\kappa_{m}} s .
$$

It turns out that the same approximation can also be derived from the tQSSA. Note that this solution does not satisfy the initial condition $c(0)=0$, and is only valid in the quasi-steady state. We call this approximation an outer solution. Substituting the dimensionless variables and (6) into equation (2) gives

$$
\frac{\mathrm{ds}}{\mathrm{dT}}=-\frac{\mathrm{E}_{0}+\mathrm{S}_{0}+\kappa_{\mathrm{m}}}{\mathrm{E}_{0}+\mathrm{S}_{0} \mathrm{~s}+\mathrm{K}_{\mathrm{m}}} \mathrm{s}, \quad \mathrm{s}(0)=1 .
$$

Solving equation (7) gives the outer solution of $s, s_{\mathrm{O}}$, in the form

$$
\left(E_{0}+\kappa_{m}\right) \ln s_{O}(T)+S_{0}\left(s_{O}(T)-1\right)+\left(E_{0}+S_{0}+K_{m}\right) T=0
$$

which is also the same as the solution obtained via tQSSA.

\subsection{Inner solution}

Since the outer solutions do not satisfy the initial conditions, we presume there is another set of early time solutions in an initial or pre-steady state layer. Here the solutions are called inner solutions.

Consequently, a new rate equation is derived for the substrate concentration upon substitution of the dimensionless variables $s$ and $\tau$ into equa- 
tion (2):

$$
\frac{d s}{d \tau}=-\frac{\kappa_{2} E_{0}}{\kappa_{1}\left(E_{0}+S_{0}+\kappa_{m}\right)^{2}} c, \quad s(0)=1
$$

One sees that as $\epsilon \rightarrow 0$, equation (9) becomes $\frac{\mathrm{d} s}{\mathrm{~d} \tau}=0$ and so $s$ is approximately constant throughout the pre-steady state. After imposing the initial condition, the inner solution (represented by subscript ' $\mathrm{I}$ ') in this region is

$$
\mathrm{s}_{\mathrm{I}}(\tau)=\mathrm{s}(0)=1
$$

On the other hand, insert the scaled variables into equation (3) and replace $s=1$, the rate equation of complex is reformulated as

$$
\frac{d c}{d \tau}=1-c-\gamma c^{2}, \quad c(0)=0 .
$$

Solving equation (11) gives the inner solution of complex concentration

$$
c_{I}(\tau)=\frac{2[\exp (\sqrt{4 \gamma-1} \tau)-1]}{(1-\sqrt{1-4 \gamma}) \exp (\sqrt{4 \gamma-1} \tau)-(1+\sqrt{1-4 \gamma})}
$$

which satisfies the actual biochemical phenomena, that is, no complex concentration appears at the beginning; in other words, the initial condition $c(0)=0$ holds by the inner solution.

\subsection{Matching and uniform approximation}

The inner solution, valid in the transient period, together with the outer solution, valid in the post-transient period, comprise a total solution for the system. These solutions have a common limit or overlap term, that is, where the outer solution begins to take over from inner solution. 


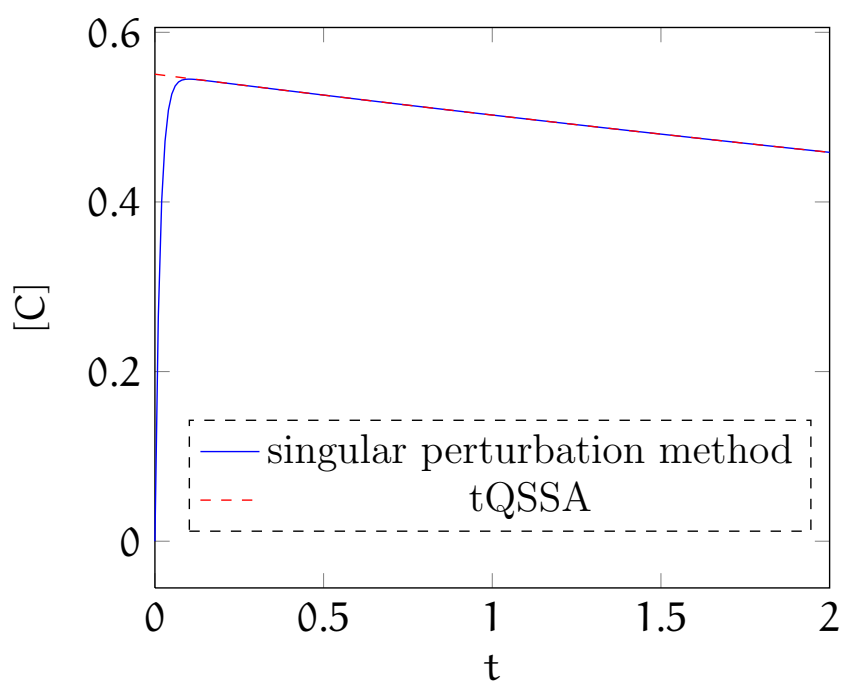

Figure 1: The computed complex concentrations with $E_{0}=200, S_{0}=180$, $\mathrm{K}_{1}=0.001, \mathrm{~K}_{2}=30$ and $\mathrm{K}_{-1}=35$. 
Consider now $\epsilon \rightarrow 0, \tau \rightarrow \infty$ and $\mathrm{T} \rightarrow 0$ respectively. The common limit of the inner and outer solutions is defined as

$$
\lim _{\epsilon \rightarrow 0}\left[\left.y_{O}(T)\right|_{T=0}\right]=\lim _{\epsilon \rightarrow 0}\left[\left.y_{I}(\tau)\right|_{\tau=\infty}\right] .
$$

In other words, the inner and outer solutions are matched if in the limit of $\epsilon \rightarrow 0$, the inner solution as $\tau \rightarrow \infty$ is equal to the outer solution as $T \rightarrow 0$.

Lastly, the final solution, the so-called uniform approximation is obtained by adding the inner and outer solutions and subtracting their common part.

Now, let us apply the matching condition to the outer and inner solutions of the substrate concentration, that is, to equations (8) and (10). We have

$$
\lim _{\epsilon \rightarrow 0}\left[\left.s_{O}(T)\right|_{T=0}\right]=\lim _{\epsilon \rightarrow 0}\left[\left.s_{I}(\tau)\right|_{\tau=\infty}\right]=1 .
$$

On the other hand, checking the limit of the outer and inner solutions of the complex gives

$$
\lim _{\epsilon \rightarrow 0}\left[\left.\mathrm{c}_{\mathrm{O}}(\mathrm{T})\right|_{\mathrm{T}=0}\right]=\lim _{\epsilon \rightarrow 0}\left[\left.\mathrm{c}_{\mathrm{I}}(\tau)\right|_{\tau=\infty}\right] \approx 2(1+\sqrt{1-4 \gamma})^{-1} .
$$

The uniform approximations for $\mathrm{s}$ and $\mathrm{c}$ are eventually derived as

$$
\begin{aligned}
& s_{\mathrm{u}}=s_{\mathrm{O}}+\mathrm{s}_{\mathrm{I}}-1=\mathrm{s}_{\mathrm{O}}, \\
& \mathrm{c}_{\mathrm{u}}=\mathrm{c}_{\mathrm{O}}+\mathrm{c}_{\mathrm{I}}-2(1+\sqrt{1-4 \gamma})^{-1} .
\end{aligned}
$$

Through equation (12), we find that the approximations obtained via tQSSA and its singular perturbation procedures are exactly the same, thus tQSSA is considered as a reasonable approximation to the substrate concentration. On the other hand, the singular perturbation analysis has successfully corrected the defect of tQSSA; that is, the initial condition is satisfied by introducing the inner solution, $\mathrm{c}_{\mathrm{I}}$ (refer Figure 1). 


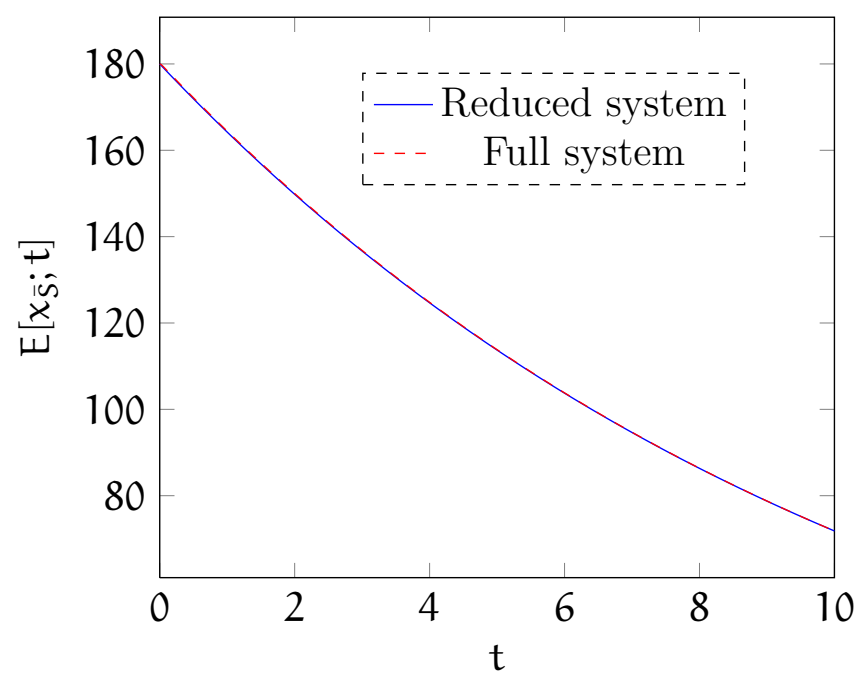

FiguRE 2: Expected value of total substrate computed with $x_{e_{0}}=200$, $x_{s_{0}}=180, \kappa_{1}=0.001, \kappa_{2}=30$ and $\kappa_{-1}=35$, via two approaches: full system of CME and the reduced system, namely the CME in conjunction with tQSSA.

\section{Applying the tQSSA to the chemical master equation}

In molecular biology, stochasticity is an important driver. Here, we consider the Michaelis-Menten mechanism in reaction (1) where we initially have $x_{e_{0}}$ copies of enzyme, $x_{s_{0}}$ copies of the total substrate and zero copies of complex and product. The probability of a state $X=\left(x_{c}, x_{\bar{S}}\right)$ at time $t$ is denoted by $\operatorname{Pr}\left(x_{\mathfrak{c}}, x_{\bar{s}} ; t\right)$ and is governed by the chemical master equation $(\mathrm{CME})$ :

$$
\frac{d \operatorname{Pr}(X ; t)}{d t}=\sum_{i=1}^{3} \alpha_{i}\left(X-z_{i}\right) \operatorname{Pr}\left(X-z_{i} ; t\right)-\alpha_{i}(X, t) \operatorname{Pr}(X ; t) .
$$




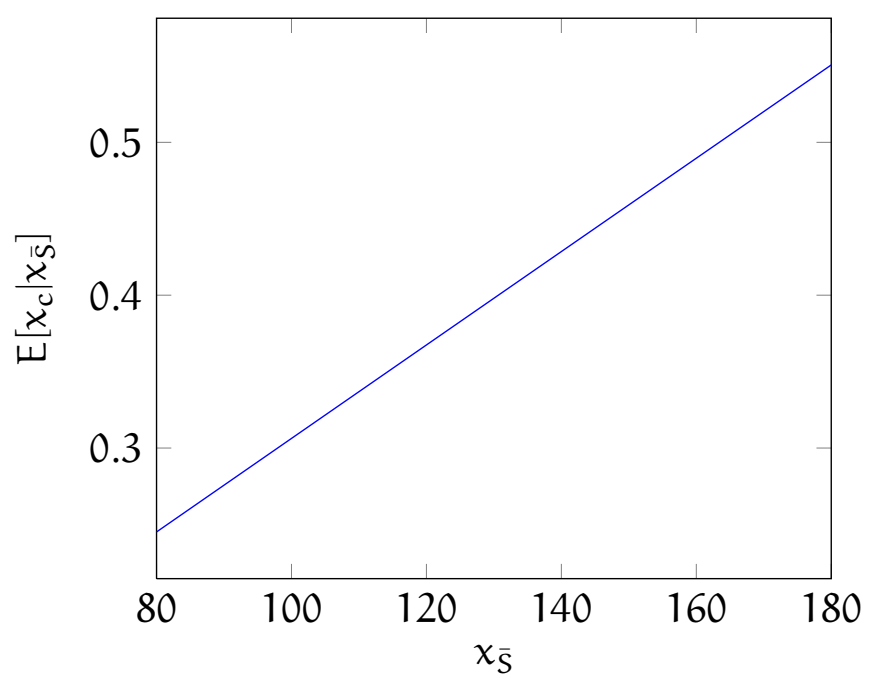

Figure 3: The conditional expectation of the complex level, $E\left[x_{\mathfrak{c}} \mid x_{\bar{S}}\right]$, obtained through equations (15) and (16).

Here, $\alpha_{i}$ is the ith reaction's propensity: $\alpha_{1}=k_{1}\left(x_{e_{0}}-x_{c}\right)\left(x_{\bar{s}}-x_{c}\right), \alpha_{2}=\kappa_{2} x_{c}$ and $\alpha_{3}=\kappa_{-1} x_{c}$. Furthermore, $z_{i}$ is the ith reaction's stoichiometric vector: $z_{1}=[1,0]^{\top}, z_{2}=[-1,-1]^{\top}$ and $z_{3}=[-1,0]^{\top}$.

According to Goutsias [6], the conditional probability $\operatorname{Pr}\left(x_{\mathbf{c}} \mid x_{\bar{S}} ; t\right)$ approximately solves

$$
\begin{aligned}
\frac{d \operatorname{Pr}\left(x_{c} \mid x_{\bar{S}} ; t\right)}{d t}= & -\left(\kappa_{1}\left(x_{e_{0}}-x_{c}\right)\left(x_{\bar{S}}-x_{c}\right)+\left(\kappa_{-1}+\kappa_{2}\right) x_{c}\right) \operatorname{Pr}\left(x_{c} \mid x_{\bar{S}} ; t\right) \\
& +\kappa_{1}\left(x_{e_{0}}-x_{c}+1\right)\left(x_{\bar{S}}-x_{c}+1\right) \operatorname{Pr}\left(x_{c}-1 \mid x_{\bar{S}} ; t\right) \\
& +\left(\kappa_{-1}+\kappa_{2}\right)\left(x_{c}+1\right) \operatorname{Pr}\left(x_{c}+1 \mid x_{\bar{S}} ; t\right) .
\end{aligned}
$$

Recall that the tQSSA leads to the DAE system (4), and, in particular, a constant $c_{O}$ for any given $s_{O}$. Following an idea of Cao et al. [4], this suggests that for a given $x_{\bar{S}}$ the conditional probability $\operatorname{Pr}\left(x_{\mathfrak{c}} \mid x_{\bar{S}}\right)$ is stationary, that 
TABLE 1: Comparison of the computations of the full and reduced systems.

\begin{tabular}{lrrrr}
\hline Limiting factor, $\chi_{s_{0}}$ & 90 & 120 & 150 & 180 \\
\hline Computation time for full system $(\mathrm{s})$ & 392 & 609 & 1261 & 2430 \\
Computation time for reduced system $(\mathrm{s})$ & 5.0 & 5.9 & 7.3 & 8.4 \\
Ratio & 78 & 103 & 173 & 289 \\
Percent relative error of $\mathrm{E}\left[\mathrm{x}_{\overline{\mathrm{S}}}\right](\%)$ & 0.14 & 0.14 & 0.14 & 0.14 \\
\hline
\end{tabular}

is

$$
\frac{d \operatorname{Pr}\left(x_{c} \mid x_{\bar{S}} ; t\right)}{d t}=0 .
$$

It follows that $\operatorname{Pr}\left(x_{c} \mid x_{\bar{s}} ; t\right)$ can be found by solving the homogeneous system obtained by replacing the left hand side of equation (15) by zero.

On the other hand, summing the CME (14) over $x_{c}$ and replacing $\operatorname{Pr}\left(x_{c}, x_{\bar{S}} ; t\right)$ by $\operatorname{Pr}\left(x_{\mathbf{c}} \mid x_{\bar{S}}\right) \operatorname{Pr}\left(x_{\bar{S}} ; t\right)$ in the CME gives

$$
\frac{d \operatorname{Pr}\left(x_{\bar{S}} ; t\right)}{d t}=\kappa_{2} E\left[x_{c} \mid x_{\bar{S}}+1\right] \operatorname{Pr}\left(x_{\bar{S}}+1 ; t\right)-\kappa_{2} E\left[x_{c} \mid x_{\bar{S}}\right] \operatorname{Pr}\left(x_{\bar{S}} ; t\right) .
$$

Note that once $\operatorname{Pr}\left(x_{c} \mid x_{\bar{S}} ; t\right)$ and, hence, $E\left[x_{c} \mid x_{\bar{S}}\right]$ have been determined, the CME (17) is of lower dimension than the original CME (14).

\section{Discussion}

We revisited the tQSSA for simple enzymatic reactions and derived the reduced equations using a perturbation ansatz. While we mainly do this for the deterministic case we also derive a model for the stochastic case.

The application of the reduced system obtained using this approach, equations (16) and (17), leads to substantial computational savings, and the results obtained match favorably with the results of the full system, by equation (14), see Figures 2 and 3 and Table 1 . Since $x_{s_{0}}$ is the limiting 
factor [3] of the system, the sizes of the propensity matrices of full and reduced systems are $x_{s_{0}}^{2} \times x_{s_{0}}^{2}$ and $x_{s_{0}} \times x_{s_{0}}$ respectively. Our computations (see Table 1), shown that the computation time grows exponentially for the full system but linearly for the reduced system. The relative error computed for the expected value of the total substrate turns out to be around $10^{-3}$. The justification of the application of tQSSA to the CME will be discussed in future work.

Acknowledgements: The authors thank Professor Andrew Bassom for his kind advice.

\section{References}

[1] G. A. Baker and P. R. Graves-Morris. Pade Approximants (Encyclopedia of Mathematics and its Application). Cambridge University Press, 1984. C435

[2] J. A. M. Borghans, R. J. d. Boer, and L. A. Segel. Extending the quasi-steady state approximation by changing variables. Bulletin of Mathematical Biology, 58:43-63, 1996. doi:10.1007/BF02458281. C431, C432, C434

[3] G. E. Briggs and J. B. S. Haldane. A note on the kinetics of enzyme action. The Biochemical Journal, 19(2):338-339, 1925. http://www . biochemj . org/bj/019/0338/bj0190338_browse.htm. C431, C442

[4] Y. Cao, D. T. Gillespie, and L. R. Petzold. The slow-scale stochastic simulation algorithm. The Journal of Chemical Physics, 122:014116-1-18, 2005. doi:10.1063/1.1824902. C440 
[5] S. Cha and Cha C. M. Kinetics of cyclic enzyme systems. Molecular Pharmacology, 1:178-189, 1965. http://molpharm. aspetjournals.org/cgi/reprint/1/2/178. C435

[6] J. Goutsias. Quasi equilibrium approximation of fast reaction kinetics in stochastic biochemical systems. Journal of Chemical Physics, 122:184102-1-15, 2005. doi:10.1063/1.1889434. C440

[7] L. Michaelis and M. Menten. Die kinetik der invertinwirkung. Biochemische Zeitschrift, 49:333-369, 1913. C430

[8] J. M. Nelson and H. W. Larson. Kinetics of invertase action. The Journal of Biological Chemistry, 73(11):223-236, 1927. http://www.jbc.org/cgi/reprint/73/1/223. C430

[9] L. A. Segel. On the validity of the steady state assumption of enzyme kinetics. Bulletin of Mathematical Biology, 50:579-593, 1988. doi:10.1007/BF02460092. C431, C432

[10] A. N. Tikhnov. Systems of differential equations containing a small parameter multiplying the derivatives. Mat. Sb., 31:575-586, 1952. C433

\section{Author addresses}

1. C. F. Khoo, Mathematical Sciences Institute, Australian National University, Canberra, Australia. mailto:khoo@maths . anu.edu . au

2. M. Hegland, Mathematical Sciences Institute, Australian National University, Canberra, Australia. 